(c) IEEE, 2010. This is the author's version of the work. Personal use of this material is permitted. However, permission to reprint/republish this material for advertising or promotional purpose or for creating new collective works for resale or redistribution to servers or lists, or to reuse any copyrighted component of this work in other works must be obtained from the IEEE.

The definite version is published in Proc. International Conference on Information Fusion (FUSION 2007), Quebec, Canada 2007.

\title{
Analysis of Sensor and Fusion Schedules of a Time-Triggered Sensor Fusion System
}

\author{
Moritz Mauthner Wilfried Elmenreich Richard Altendorfer Alexander Kirchner
}

\begin{abstract}
In this paper we analyze the sensor and fusion schedules of a time-triggered, Kalman filter based, multi-sensor fusion system. The fusion system is used as an environmental perception platform for advanced driver assistance systems and delivers its service to a safety related application. As the application demands cyclic updates with bounded accuracy, the influence of the sensor and fusion schedules on the service accuracy is analysed, which enables us to optimize the system without model dependent simulations.
\end{abstract}

\section{INTRODUCTION}

Multi-sensor data fusion systems are the enabling technology for advanced driver assistance systems. New features like adaptive cruise control with active steering, lane departure alert, parking pilot, and automatic emergency brake will be standard in the next car generation as electronic stabilization systems and anti lock braking systems are at present.

This paper deals with the optimization of a time-triggered, Kalman filter based, sensor fusion system for driver assistance systems consisting of two sensors, a bus system and a fusion/prediction core. It tracks objects and is supposed to deliver a real time image (model) of the environment at fixed intervals with bounded accuracy to a safety related application.

The rest of the paper is structured as follows: In section II the system set-up is described. In section III out of sequence measurement (OOSM) treatment strategies are discussed. Section IV discusses the overall system response time. We analyse the system schedule in section V. In section VI a conclusion is drawn.

\section{SYSTEM DESCRIPTION}

We consider a system with two identical sensors that measure the states of surrounding objects and transmit this information via a time-triggered bus system to a fusion/prediction core. The fusion/prediction core fuses the incoming information and then predicts the fused object states to real time as demanded by the safety related application (see figure 1). We use the following definitions:

- the cycle time $t_{C}$ of a process is the time interval that this process requires for completion of its service while the start of two consecutive processes is $t_{C}$ apart

- the execution time $t_{T}$ of a process is the time interval in which this process completes its service where the start of two consecutive processes can be more than $t_{T}$ apart

M. Mauthner is with Volkswagen AG, Wolfsburg, Germany moritz.mauthner@volkswagen. de

W. Elmenreich is with the Department of Computer Engineering, Vienna University of Technology, Vienna Austria wilevmars.ac.at

R. Altendorfer is with Audi Electronics Ventures GmbH, Ingolstadt, Germany richard.altendorfereaudi.de

A. Kirchner is with Volkswagen AG, Wolfsburg, Germany alexander.kirchner@volkswagen. de
- the phase $t_{P}$ of a process is defined as the time interval between the start of this process relative to the start of the first cycle of sensor 1

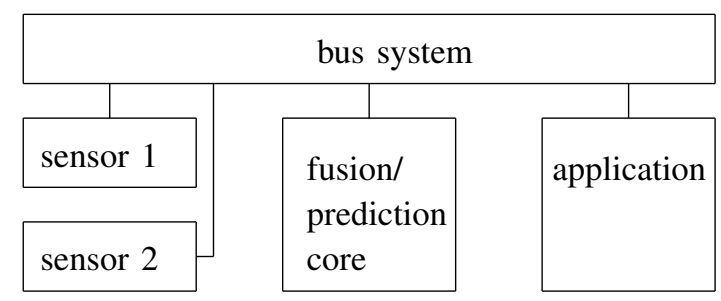

Fig. 1. Sensor fusion system

The two sensors have cycle times $t_{C}^{\text {sens } 1}$ and $t_{C}^{\text {sens } 2}$ that are identical to their preprocessing times and therefore adjustable by the choice of the speed of the sensor's processor. They deliver their measurement states $\vec{z}_{t_{k}}^{\text {sens } 1}$ and $\vec{z}_{t_{k}}^{\text {sens } 2}$ (with constant covariance matrices $R_{t_{k}}^{\text {sens } 1}$ and $R_{t_{k}}^{\text {sens } 2}$ ) over a time-triggered bus system to a fusion/prediction core. The bus system uses time division multiple access (TDMA) with fixed cycle time $t_{C}^{t t a}$ (the bus is not exclusively used by the sensor fusion system, hence the cycle time is assumed to be determined by external constraints). At the fusion/prediction core the information is processed in order to establish a robust image of the environment.

In figure 2 all cycle times $t_{C}$, process execution times $t_{T}$ and phases $t_{P}$, relative to sensor 1 , of sensor preprocessing $\left(t_{C}^{\text {sens } 1}, t_{C}^{\text {sens } 2}, t_{P}^{\text {sens }}\right)$, measurement transmission $\left(t_{C}^{t t a}, t_{P}^{t t a 1}, t_{P}^{t t a 2}\right)$ and generation of the application update $\left(t_{C}^{\text {pre }}, t_{T}^{\text {pre }}\right.$ and $\left.t_{P}^{\text {pre }}\right)$ that are processed within the fusion system are visualized.

To process the incoming measurements at the fusion/prediction core, we use a Kalman filter approach as described in [1]. As the Kalman filter is a widely used and well known algorithm for tracking moving targets, we will only give a short overview of the notation used throughout this paper. The estimated state vector of the tracked object $\hat{\vec{x}}\left(t_{k-1} \mid t_{k-1}\right)$ is updated with measurements at discrete points in time $t_{k}$. The state prediction from $t_{k-1}$ to $t_{k}$ is given by $\hat{\vec{x}}\left(t_{k} \mid t_{k-1}\right)=F\left(t_{k}-t_{k-1}\right) \cdot \hat{\vec{x}}\left(t_{k-1} \mid t_{k-1}\right)$. The predicted state covariance matrix is computed as $P\left(t_{k} \mid t_{k-1}\right)=F\left(t_{k}-t_{k-1}\right)$. $P\left(t_{k-1} \mid t_{k-1}\right) \cdot\left(F\left(t_{k}-t_{k-1}\right)\right)^{T}+Q_{t_{k-1}}$. The measurement update of the predicted system state is $\hat{\vec{x}}\left(t_{k} \mid t_{k}\right)=\hat{\vec{x}}\left(t_{k} \mid t_{k-1}\right)+$ $K_{t_{k}} \cdot \vec{v}_{t_{k}}$ with $K_{t_{k}}=P\left(t_{k} \mid t_{k-1}\right) \cdot H_{t_{k}}^{T} \cdot\left(H_{t_{k}} P\left(t_{k} \mid t_{k-1}\right) H_{t_{k}}^{T}+R_{t_{k}}\right)^{-1}$, $\vec{v}_{t_{k}}=\vec{z}_{t_{k}}-\hat{\vec{z}}_{t_{k}}$ and $\hat{\vec{z}}_{t_{k}}=H_{t_{k}} \cdot \hat{\vec{x}}\left(t_{k}\right)$. The update of the covariance matrix is given by $P\left(t_{k} \mid t_{k}\right)=\left(I-K_{t_{k}} \cdot H_{t_{k}}\right) \cdot P\left(t_{k} \mid t_{k-1}\right) \cdot$ $\left(I-K_{t_{k}} \cdot H_{t_{k}}\right)^{T}+K_{t_{k}} \cdot R_{t_{k}} \cdot K_{t_{k}}^{T}$. The fusion/prediction core processes a measurement within $t_{T}^{f u s}$. 


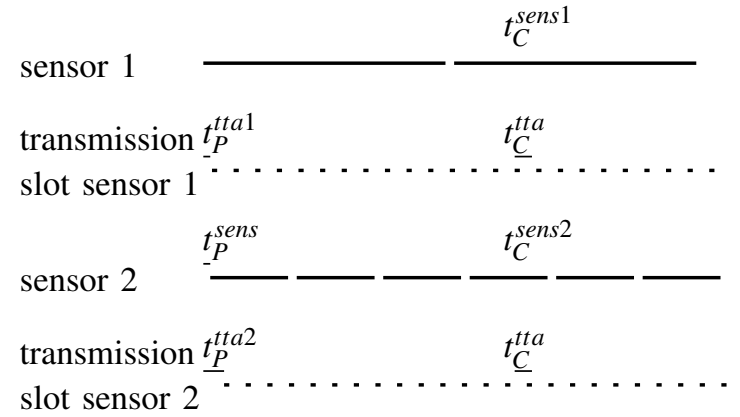

slot for

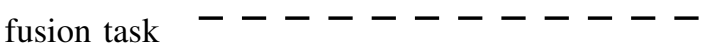

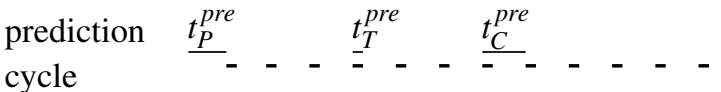

real time

Fig. 2. System schedule

\section{Out-OF-Sequence Measurement Treatment}

In multi-sensor tracking systems, information about the same object can arrive out of sequence [2], [3], that means not in chronological order. Often, OOSM behavior is caused by an indeterministic transmission system, where the transmission time of a message may vary so much that a message from a later measurement may overtake a newer measurement. Such behavior is caused by transmission protocols with many retries such as many Internet protocols (e. g., TCP/IP) or in networks with dynamic routing (Internet, wireless sensor networks).

However, even if communication protocols with deterministic behavior, such as time-triggered approaches like flexray [4], TTCAN [5], TTP [6], or TTP/A [7] are used, the OOSM problem may arise.

Figure 3 depicts a situation with an OOSM problem that is independent from communication system issues. Due to different preprocessing times the indicated measurement from sensor 2 is received earlier at the fusion core than the indicated measurement from sensor 1 although the measurement from sensor 2 is newer.

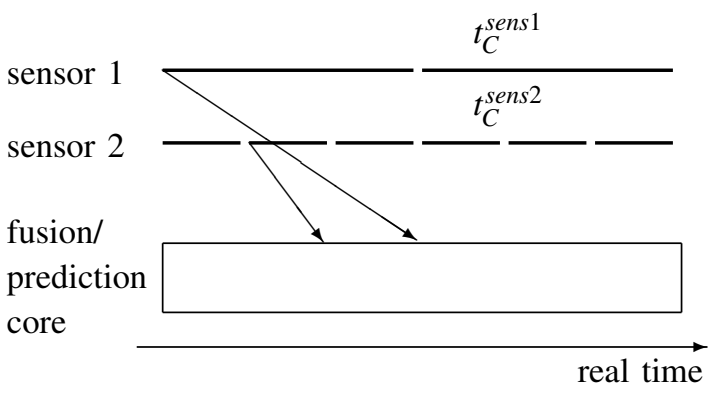

Fig. 3. Origin of out-of-sequence measurements

As the Kalman filter in the form of [1] can not handle OOSMs, architectural and algorithmic solutions have been developed.

Kaempchen et al. [8] use a measurement buffer between bus and fusion/prediction core. No measurement is delivered to the fusion/prediction core, before all measurements that have been sampled earlier have arrived at the buffer. Within the buffer the measurements are sorted chronologically. Kaempchen et al. further discuss the maximum latency (here defined as the time difference between the instant of the composition of the image representing the surrounding environment provided by sensor fusion and the bygone instant where this image was true) that arises between measurement recording and measurement fusion. It is distinguished between situations where only knowledge of the maximum measurement cycle times and situations where full knowledge of the measurement cycle times is available.

Another way to solve the OOSM problem are special algorithms which will be called "advanced algorithms" throughout this paper. These algorithms deal with one-lag and multi-lag delays, filtering and tracking, linear and non-linear systems as well as single-model and multi-model approaches [9], [10], [11], [12], [13], [14], [15], [16], [17], [18] and enable us to use the information of the faster sensor as soon as it is delivered to the fusion/prediction core.

For sake of discussion we will only consider the buffering approach as presented in [8] referred to as $B U F F$ and the OOSM treatment algorithm All of [10] referred to as $A D V A$.

\section{System Response Time}

As the application is safety related, it expects to periodically receive (cycle time $t_{C}^{\text {pre }}$ ) real time object states with bounded accuracy. Due to the fact that the fused states always lag behind real time irrespective of the specific OOSM treatment applied, this image of the environment has to be predicted from state time $t_{S T}$ to real time $t_{R T}$. The state time is the current time of the Kalman filter state and coincides with the measurement time of the latest fused (non-OOSM) sensor measurement, whereas real time is the actual physical time. The real time covariance matrix of the state can be determined by predicting the state time covariance matrix from state time $t_{S T}$ to real time $t_{R T}$ by $P\left(t_{R T} \mid t_{S T}\right)=F\left(t_{R T}-t_{S T}\right) \cdot P\left(t_{S T} \mid t_{S T}\right)$. $\left(F\left(t_{R T}-t_{S T}\right)\right)^{T}+Q_{S T}$.

As the dependence of $F\left(t_{R T}-t_{S T}\right)$ and $Q_{S T}$ on the prediction interval is well known for a given system, it is thus possible to consider the effect of the system parameters and the strategy for the treatment of OOSM (see also section III) on the overall system response time $\left(t_{R T}-t_{S T}\right)$ as well as state time and real time covariance matrices of the state $\left(P\left(t_{S T} \mid t_{S T}\right)\right.$ and $P\left(t_{R T} \mid t_{S T}\right)$ ).

With the overall system response time as $\Delta t=t_{R T}-t_{S T}$, $\operatorname{det}\left(P\left(t_{R T} \mid t_{S T}\right)\right)$ is given by

$$
\begin{array}{r}
\operatorname{det}\left(P\left(t_{R T} \mid t_{S T}\right)\right)= \\
\operatorname{det}\left(F(\Delta t) \cdot P\left(t_{S T} \mid t_{S T}\right) \cdot F(\Delta t)^{T}+Q_{S T}\right)= \\
\operatorname{det}\left(P\left(t_{S T} \mid t_{S T}\right)\right)+\Delta t \cdot P\left(t_{S T} \mid t_{S T}\right)(1,1) \cdot q+ \\
O\left(\Delta t^{2}\right)
\end{array}
$$

This allows us to separate the influence of the sensor schedules and the OOSM treatment, which affect the state time covariance matrix $P\left(t_{S T} \mid t_{S T}\right)$, and the influence of the bus, fusion and prediction schedules that affect the system 
response time and such the covariance at real time $P\left(t_{S T} \mid t_{S T}\right)$. From eq. 1 we see that, given $\operatorname{det}\left(P\left(t_{S T} \mid t_{S T}\right)\right)$, the leading order increase of the covariance area at real time is linear in $\Delta t$. As will be demonstrated in section $\mathrm{V}$, the variations in $P\left(t_{S T} \mid t_{S T}\right)$ are negligible and it then suffices to minimize $\Delta t$ in order to maximize the fusion accuracy at real time.

\section{System Schedule}

First we simulate the entire system at a given point in schedule parameter space. We use a simple dynamic system and measurement model in the fusion/prediction core (see appendix) and employ a Kalman filter (by linearization around a setpoint) as opposed to an extended Kalman filter as our estimation algorithm. As the computation of the covariance matrices of a Kalman filter is completely independent of the state and measurement vectors we need not input specific measurements and are thus independent of the actual driving situation. After convergence of the Kalman filter ${ }^{1}$ the maximum of $\operatorname{det}\left(P\left(t_{R T} \mid t_{S T}\right)\right)$ is recorded. This constitutes one iteration of the optimization (minimization of $\operatorname{det}\left(P\left(t_{R T} \mid t_{S T}\right)\right)$ ) over the regions of the schedule parameter space and we will discuss both the area of the covariance ellipse at state time $\operatorname{det}\left(P\left(t_{S T} \mid t_{S T}\right)\right)$ as well as at real time $\operatorname{det}\left(P\left(t_{R T} \mid t_{S T}\right)\right)$.

As $t_{C}^{\text {tta }}$ and $t_{C}^{\text {pre }}$ are fixed (see sections II and IV), $t_{C}^{\text {sens } 1}$, $t_{C}^{\text {sens } 2}, t_{T}^{f u s}$ and $t_{T}^{\text {pre }}$ are the only system parameters whose modification changes the hardware of the system and affects such the overall system costs. In contrast, $t_{P}^{\text {sens }}, t_{P}^{t t a 1}, t_{P}^{t t a 2}$ and $t_{P}^{p r e}$ can be modified without affecting the costs of the system by simply changing software parameters. We assume $t_{C}^{t t a}$ to be sufficiently small and will neglect $t_{C}^{t t a}, t_{P}^{t t a 1}$ and $t_{P}^{t t a 2}$ in the further. That means that the preprocessed information is transmitted without further delay from the sensors to the fusion core. For every configuration of $t_{C}^{\text {sens } 1}, t_{C}^{\text {sens } 2}, t_{T}^{f u s}$ and $t_{T}^{\text {pre }}$, we choose $t_{P}^{\text {sens }}$ and $t_{P}^{\text {pre }}$ that minimize the maximum of $\operatorname{det}\left(P\left(t_{R T} \mid t_{S T}\right)\right)$ which has been computed numerically over a simulation time of 10 seconds $\left(t_{\operatorname{Sim}}=10 \mathrm{~s}\right)$ performed with a simple dynamic system and measurement model (see appendix). $t_{T}^{\text {pre }}$ is approximated by $t_{T}^{\text {pre }} \approx \frac{t_{T}^{f u s}}{3}$. Note that all temporal parameters are varied in $1 \mathrm{~ms}$ steps.

In figure 4 we visualize the state time and real time accuracy for a given point in parameter space. We perceive, that the difference between the buffering and advanced algorithms approach for state time accuracy is negligible in comparison to the effects that are caused by prediction from state time to real time. Due to this the accuracy optimization can be achieved by evaluation of the minimum (by variation of the parameter space variables) of the maximum time difference between state time and real time that occurs within one macro period (least common multiple of all cycle-times) after the Kalman filter has converged.

For the buffering approach, we can describe the maximum of the interval $t_{R T}-t_{S T}$ (see figure 5) as maximum of the series $t_{R T-S T \quad l, m}^{B U F F}$

\footnotetext{
${ }^{1}$ Convergence of the Kalman filter here means that the covariance matrices have assumed a regular, periodic pattern. A constant steady state covariance matrix cannot be expected since the Kalman filter receives measurements from different sensors at different times.
}

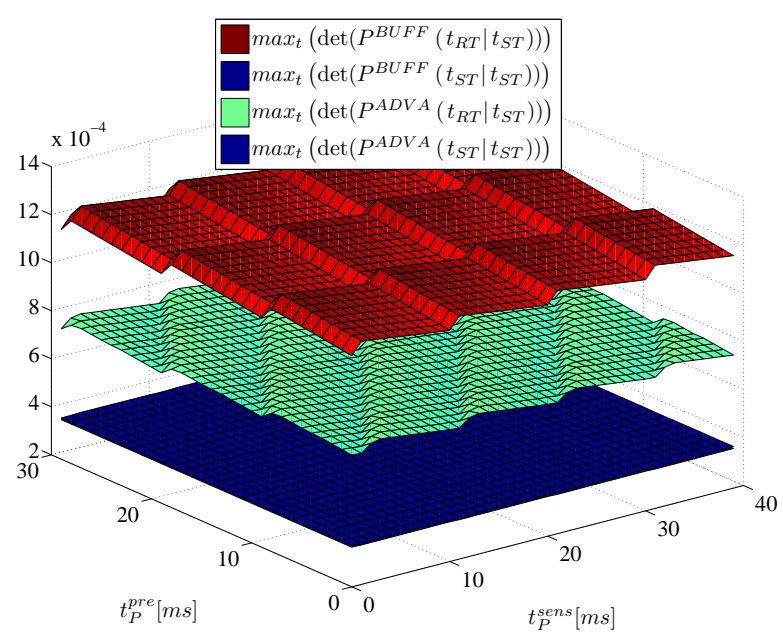

Fig. 4. State time and real time accuracy for $t_{C}^{\text {sens } 1}=130 \mathrm{~ms}, t_{C}^{\text {sens } 2}=40 \mathrm{~ms}$

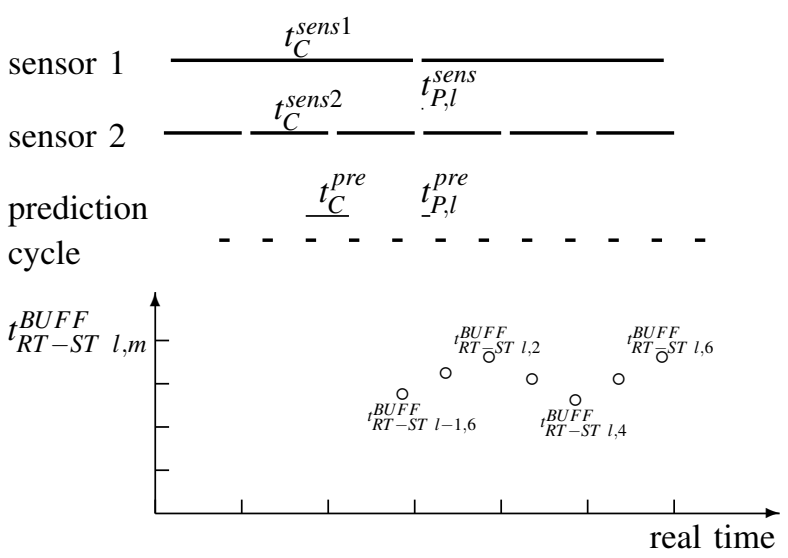

Fig. 5. $t_{R T-S T l, m}^{B U F}$ profile

$$
t_{R T-S T \quad l, m}^{B U F F}=\left\{\begin{array}{l}
(m-1) t_{C}^{\text {pre }}+t_{T}^{\text {pre }}+t_{P, l}^{\text {pre }}+t_{C}^{\text {sens } 1}+ \\
(1-n)\left(t_{C}^{\text {sens } 2}-t_{P, l-1}^{\text {sens }}\right) \\
\text { if } 0 \leq n<2 \\
(m-1) t_{C}^{\text {pre }}+t_{T}^{\text {pre }}+t_{P, l}^{\text {pre }}+ \\
t_{C}^{\text {sens } 1}+(2-n) t_{C}^{\text {sens } 2}-t_{P, l-1}^{\text {sens }} \\
\text { if } 2 \leq n
\end{array}\right.
$$

with

$$
\begin{gathered}
l=1,2, \ldots,\left\lfloor\frac{t_{\text {Sim }}}{t_{C}^{\text {sens } 1}}\right\rfloor \\
m=1,2, \ldots,\left\lceil\frac{t_{C}^{\text {sens } 1}-t_{P, l}^{\text {pre }}}{t_{C}^{\text {pre }}}\right\rceil,
\end{gathered}
$$

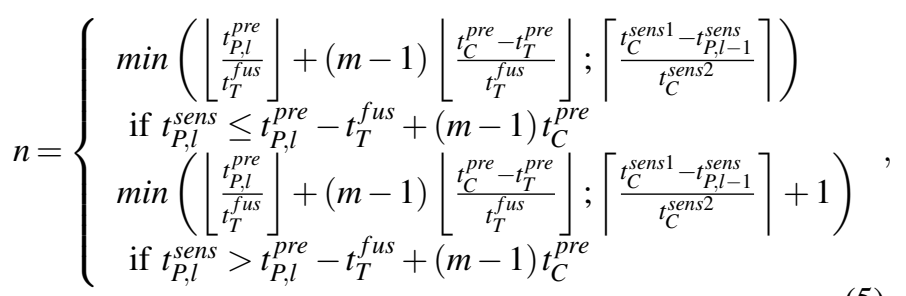




$$
t_{P, l}^{\text {sens }}=t_{P, l-1}^{\text {sens }}+\left\lceil\frac{t_{C}^{\text {sens } 1}-t_{P, l-1}^{\text {sens }}}{t_{C}^{\text {sens } 2}}\right\rceil t_{C}^{\text {sens } 2}-t_{C}^{\text {sens } 1}
$$

and

$$
t_{P, l}^{\text {pre }}=t_{P, l-1}^{p r e}+\left\lceil\frac{t_{C}^{\text {sens } 1}-t_{P, l-1}^{p r e}}{t_{C}^{p r e}}\right\rceil t_{C}^{\text {pre }}-t_{C}^{\text {sens } 1}
$$

We consider the algorithmic overhead of the advanced algorithms in comparison to the simple buffering approach by adding a penalty term to $t_{T}^{\text {fus }} t_{T}^{\text {fus ADVA }}=t_{T}^{\text {fus }}+t_{T}^{\text {overhead } 2}$ and can describe the maximum of the interval $t_{R T}-t_{S T}$ (see figure 6) as maximum of the series $t_{R T-S T}^{A D V A}$ o
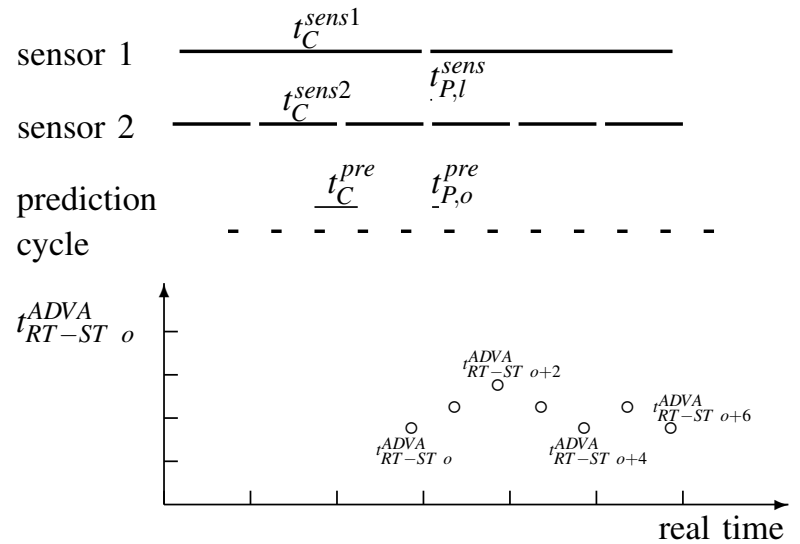

Fig. 6. $t_{R T-S T \text { o }}^{A D V A}$ profile

$$
t_{R T-S T \text { o }}^{A D V A}=t_{T}^{\text {pre }}+t_{P, o}^{p r e}+\min \left(\left\lceil\frac{t_{T}^{f u s}}{t_{P, o}^{\text {pre }}}\right\rceil ; 2\right) t_{C}^{\text {sens } 2}
$$

with

$$
o=1,2, \ldots,\left\lfloor\frac{t_{\text {Sim }}}{t_{C}^{\text {sens } 2}}\right\rfloor
$$

and

$$
t_{P, o}^{p r e}=t_{P, o-1}^{p r e}+t_{C}^{p r e}-\left\lfloor\frac{t_{P, o-1}^{p r e}+t_{C}^{p r e}}{t_{C}^{\text {sens } 2}}\right\rfloor t_{C}^{\text {sens } 2}
$$

Visualization of $\max _{t}\left(t_{R T}-t_{S T}\right)_{B U F F}=\max _{l, m}\left(\begin{array}{ll}t_{R T-S T}^{B U F F} & l, m\end{array}\right)$ and $\max _{t}\left(t_{R T}-t_{S T}\right)_{A D V A}=\max _{o}\left(t_{R T-S T}^{A D V A}\right.$ o $)$ in figure 7 and comparison to figure 4 confirmes the connection between minimal prediction interval from state time to real time and optimal worst-case accuracy as stated above.

\section{CONCLUSION}

We have performed the optimization of a sensor fusion system consisting of two sensors, a bus system and a fusion/prediction core with respect to the schedule parameter space $\left(t_{C}^{\text {sens } 1}, t_{C}^{\text {sens } 2}, t_{P}^{\text {sens }}, t_{C}^{\text {pre }}, t_{T}^{\text {pre }}\right.$ and $\left.t_{P}^{\text {pre }}\right)$. The area of the covariance ellipse was chosen as the optimization criterion. We have shown that by a judicious choice of selected schedule

$$
{ }^{2} t_{T}^{\text {Overhead }} \text { was empirically determined as } t_{T}^{\text {Overhead }} \approx t_{T}^{\text {fus }} \text {. }
$$

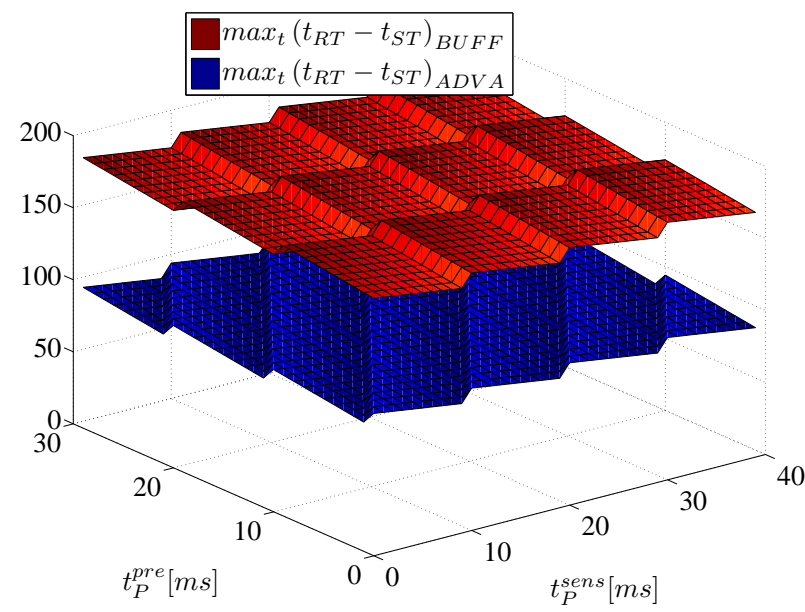

Fig. 7. $t_{R T}-t_{S T}[\mathrm{~ms}]$ for $t_{C}^{\text {sens } 1}=130 \mathrm{~ms}, t_{C}^{\text {sens } 2}=40 \mathrm{~ms}$

parameters that are adjustable at no or little cost the fusion accuracy can be improved. The time interval between the state time and the real time at which an application expects a new image of the environment was identified as the dominant factor on fusion accuracy. This means that for analytically tractable OOSM algorithms such as the buffering or advanced algorithms approach the optimization can be performed on the basis of the explicit formulae (which we have provided) without model dependent simulations.

\section{APPENDIX}

The example that is used in this paper corresponds to the one used in [10]. It is governed by the state transition matrix

$$
F\left(t_{k+1}-t_{k}\right)=\left[\begin{array}{cc}
1 & \left(t_{k+1}-t_{k}\right) \\
0 & 1
\end{array}\right]
$$

where the state consists of position and velocity of one coordinate. The dynamical system is corrupted by a zeromean, white, discretized continuous time process noise with covariance matrix $Q_{t_{k}}$

$$
Q_{t_{k}}=\left[\begin{array}{cc}
\frac{\left(t_{k+1}-t_{k}\right)^{3}}{3} & \frac{\left(t_{k+1}-t_{k}\right)^{2}}{2} \\
\frac{\left(t_{k+1}-t_{k}\right)^{2}}{2} & t_{k+1}-t_{k}
\end{array}\right] \cdot q
$$

with $q=0.5 \frac{m^{2}}{s^{3}}$ being the power spectral density of the continuous time process noise. The measurement matrix for both sensors is

$$
H_{t_{k}}^{\text {sensor } 1 / 2}=\left[\begin{array}{ll}
1 & 0 \\
0 & 1
\end{array}\right]
$$

i. e. both position and velocity are assumed to be measurable. The measurement noise of both sensors is characterized by the diagonal covariance matrix

$$
R_{t_{k}}^{\text {sensor } 1 / 2}=\left[\begin{array}{cc}
1 m^{2} & 0 \\
0 & 0.1 \frac{m^{2}}{s^{2}}
\end{array}\right] .
$$




\section{REFERENCES}

[1] A. Gelb, J. Kasper, R. Nash, C. Price, and A. Sutherland. Applied Optimal Estimation. The M.I.T. Press, 1974.

[2] S. Thomopoulos and L. Zhang. Distributed filtering with random sampling and delay. Proceedings on the 27th Conference on Decision and Control, December 1988.

[3] Y. Bar-Shalom. Multitarget-Multisensor Tracking: Applications and Advances, volume 3 of Multitarget-Multisensor Tracking. Artech House, 2000.

[4] FlexRay Consortium. Flexray communications system. Protocol specification, December 2005. Version 2.1 Revision A.

[5] F. Hartwich, B. Müller, T. Führer, and R. Hugel. Time triggered communication on CAN. In Proceedings 7th International CAN Conference, Amsterdam, The Nederlands, 2000

[6] TTAGroup. Specification of the TTP/C Protocol. TTAGroup, 2003. Available at www.ttagroup.org.

[7] H. Kopetz et al. Specification of the TTP/A protocol. Research Report 61/2002, Vienna, Austria, September 2002. Version 2.00.

[8] N. Kaempchen and K. Dietmayer. Data synchronization strategies for multi-sensor fusion. In Proceedings of the IEEE Conference on Intelligent Transportation Systems, October 2003.

[9] T. D. Larsen, N. K. Poulsen, N. A. Andersen, and O. Ravn. Incorporation of time delayed measurements in a discrete-time kalman filter. Proceedings of the 37th Conference on Decision and Control, Tampa, Florida, USA, pages 3972-3977, 1998.

[10] Y. Bar-Shalom, H. Chen, and M. Mallick. One-step solution for the multistep out-of-sequence-measurement problem in tracking. IEEE Transactions on Aerospace and Electronic Systems, 40(1), January 2004.

[11] Y. Bar-Shalom and H. Chen. IMM estimator with out-of-sequence measurements. IEEE Transactions on Aerospace and Electronic Systems, 41(1), January 2005.

[12] M. Mallick and A. Marrs. Comparison of the KF and particle filter based out-of-sequence measurement filtering algorithms. 6th International Conference on Information Fusion, pages 422-430, 2003.

[13] M. Orton and A. Marrs. Particle filters for tracking with out-of-sequence measurements. IEEE Transactions on Aerospace and Electronic Systems, 41(2), April 2005.

[14] K.-S. Zhang, X. R. Li, and Y.-M. Zhu. Optimal update with outof-sequence measurements. IEEE Transactions on Signal Processing, 53(6), JUNE 2005.

[15] S. Maskel, R. Everitt, R. Wright, and M. Briers. Multi target out-ofsequence data association. Conference of Information Fusion, 2004.

[16] S. Challa, R. Evans, and X. Wang. A bayesian solution and its approximations to out-of-sequence measurement problems. Information Fusion(4):pages 185-199, 2003.

[17] X. Wang and S. Challa. Augmented state IMM-PDA for OOSM solution to maneuvering target tracking in clutter. Proceedings of Radar 2003 International Conference, pages 479-485, September 2003. Adelaide, Australia.

[18] Y. Anxi, L. Diannong, H. Weidong, and D. Zhen. A unified out-ofsequence measurements filter. IEEE International Radar Conference, pages $453-458$, May 2005 . 